The Astrophysical Journal, 563:L41-L44, 2001 December 10

(C) 2001. The American Astronomical Society. All rights reserved. Printed in U.S.A.

\title{
IDENTIFICATION OF THE OPTICAL AND QUIESCENT COUNTERPARTS TO THE BRIGHT X-RAY TRANSIENT IN NGC 6440
}

\author{
J. J. M. in 'T Zand, ${ }^{1,2}$ M. H. van Kerkwijk, ${ }^{1}$ D. Pooley, ${ }^{3}$ F. Verbunt, ${ }^{1}$ R. Wijnands, ${ }^{3,4}$ And W. H. G. Lewin ${ }^{3}$ \\ Received 2001 October 3; accepted 2001 October 30; published 2001 November 20
}

\begin{abstract}
After 3 years of quiescence, the globular cluster NGC 6440 exhibited a bright transient X-ray source turning on in 2001 August, as noted with the Rossi X-Ray Timing Explorer All-Sky Monitor. We carried out a short target-of-opportunity observation with the Chandra X-Ray Observatory and are able to associate the transient with the brightest of 24 X-ray sources detected during quiescence in 2000 July with Chandra. Furthermore, we securely identify the optical counterpart and determine that the 1998 X-ray outburst in NGC 6440 was from the same object. This is the first time that an optical counterpart to a transient in a globular cluster is securely identified. Since the transient is a type I X-ray burster, it is established that the compact accretor is a neutron star. Thus, this transient provides an ideal case to study the quiescent emission in the optical and X-ray of a transiently accreting neutron star while knowing the distance and reddening accurately. One model that fits the quiescent spectrum is an absorbed power-law plus neutron star hydrogen atmosphere model. We find an intrinsic neutron star radius of $17_{-12}^{+31} \mathrm{~km}$ and an unabsorbed bolometric luminosity for the neutron star atmosphere of $(2.1 \pm 0.8) \times 10^{33} \mathrm{ergs} \mathrm{s}^{-1}$ that is consistent with predictions for a cooling neutron star.
\end{abstract}

Subject headings: globular clusters: individual (NGC 6440) — stars: neutron — X-rays: binaries

\section{INTRODUCTION}

Currently, 13 bright X-ray sources are known to inhabit 12 Galactic globular clusters (for a recent overview, see Sidoli et al. 2001; the 13th source was recently discovered by White \& Angelini 2001). They are low-mass X-ray binaries (LMXBs) of which at least 11 harbor a neutron star. Four are transient in nature: those in Liller 1, Terzan 5 and 6, and NGC 6440 (one could argue that Terzan 1 contains a transient source as well with an activity lasting decades; see Guainazzi, Parmar, \& Oosterbroek 1999). Thus far, none of these transients had a securely identified optical counterpart. The prospects for finding one were best for the transient in NGC 6440 because it has a relatively low reddening of $E(B-V)=1.07$ and a nonextreme distance of $8.5 \pm 0.4 \mathrm{kpc}$ (Ortolani, Barbuy, \& Bica 1994). The transient in NGC 6440 was seen in outburst in 1971 December (Markert et al. 1975; Forman, Jones, \& Tananbaum 1976) and 1998 August (in 't Zand et al. 1999; Verbunt et al. 2000). From ROSAT HRI localizations of two sources in quiescence and optical observations during the 1998 outburst, two candidate optical counterparts were identified, V1 and V2 (Verbunt et al. 2000). V2 was evaluated to be the most likely one with $B \approx 22.7$ during outburst.

Pooley et al. (2001) observed NGC 6440 for $23 \mathrm{ks}$ with the Chandra X-Ray Observatory on 2000 July 4 when the transient was quiescent. They found 24 point sources with $0.5-2.5 \mathrm{keV}$ luminosities between $1.8 \times 10^{31}$ and $9.9 \times 10^{32} \mathrm{ergs} \mathrm{s}^{-1}$, which they designate CX1-CX24. The brightest source, CX1, coincides with the optical counterpart candidate V2.

The transient in NGC 6440 erupted again in 2001 August, as detected with the All-Sky Monitor (ASM) on the Rossi X-

\footnotetext{
${ }^{1}$ Astronomical Institute, Utrecht University, P.O. Box 80 000, NL-3508 TA Utrecht, Netherlands.

${ }^{2}$ SRON National Institute for Space Research Sorbonnelaan 2, NL-3584 CA Utrecht, Netherlands; jeanz@sron.nl.

${ }^{3}$ Center for Space Research and Department of Physics, Massachusetts Institute of Technology, Cambridge, MA 02139-4307.

${ }^{4}$ Chandra Fellow.
}

Ray Timing Explorer $(R X T E) .{ }^{5}$ We proceeded to secure the identification of the optical counterpart through swift, highresolution X-ray observations, and we report the results in the present Letter. A preliminary report was already given by in 't Zand et al. (2001).

\section{OBSERVATIONS AND SOURCE IDENTIFICATION}

The ASM (Levine et al. 1996; Bradt et al. 2001) monitors NGC 6440 for 10 months of each year at a detection threshold of about 15 mcrab in the 1.5-12 keV band per day of observation. The transient exceeded this threshold during 2 weeks starting on 2001 August 2. The peak flux was $60 \pm 10$ mcrab. Continued ASM monitoring showed a reflaring episode starting on September 10, with fluxes up to $0.2 \mathrm{crab}$ and lasting for already almost 2 months at the time of this writing. The Wide Field Cameras (WFCs) on BeppoSAX (Jager et al. 1997; in 't Zand 2001) also observed NGC 6440 during this episode and detected at least 10 type I X-ray bursts. As soon as the community was alerted of the outburst (on August 13), we proposed X-ray observations with Chandra and optical observations with the European Southern Observatory's New Technology Telescope (NTT) in Director's Discretionary Time.

The Chandra (Weiskopf, O’Dell, \& van Speybroek 1996) target-of-opportunity (TOO) observation was approved and carried out on August 18.9 UT for an exposure time of $2.6 \mathrm{ks}$ with the Advanced CCD Imaging Spectrometer (ACIS; G. P. Garmire, J. A. Nousek, \& M. W. Bautz 2001, in preparation) and no grating. Despite the anticipated heavy pileup, we chose this configuration, and not the High Resolution Camera (HRC), to optimize the sensitivity such that not only the transient but also a few of the other sources in NGC 6440 would be detected (ACIS has 2 to 4 times more effective area for $0.3-8 \mathrm{keV}$ photons than HRC-I). Thus, the association of the transient with one of the quiescent sources would become independent from the Chandra attitude accuracy. Only a $2^{\prime} \times 8^{\prime}$ subarray of the back-illuminated S3 chip was read, so that the CCD

${ }^{5}$ As announced by the ASM team at http://xte.mit.edu. 
frame time was $0.8 \mathrm{~s}$ instead of $3.2 \mathrm{~s}$, and pileup effects were reduced. Figure 1 shows the obtained image, zoomed in on the globular cluster and compared with the observation during quiescence (Pooley et al. 2001). It is evident that the transient was active. Furthermore, it is closest to CX1, the brightest of the sources detected during quiescence. Based on the Chandra aspect solution for the 2001 observation (accurate to about 0 ".5) and the astrometry carried out by Pooley et al. (2001) for the 2000 observation, the angular distance between the transient and CX1 is 0.14 (the position of the transient is determined by weighing pixel position with pixel values). Of the remaining sources, CX2 and CX5 are clearly detected during the TOO observation as well, with photon rates in the full bandpass of $(8 \pm 2) \times 10^{-3}$ and $(6 \pm 1) \times 10^{-3} \mathrm{~s}^{-1}$, respectively. These rates compare well with those during the earlier observation [(7.6 \pm 0.6$) \times 10^{-3}$ and $(4.3 \pm 0.4) \times 10^{-3} \mathrm{~s}^{-1}$, respectively]. The angular distances of the transient to CX2 and CX5 are consistent with that to CX1 in the 2000 July image within $0 \prime 28 \pm 0 \prime$.'13. The distance is inconsistent with all of the other 23 sources from the 2000 July observation by at least $15 \sigma$. This confirms the transient-CX1 association.

A total of $2.6 \times 10^{3}$ events was detected within 8.0 from the source centroid. In order to estimate the flux and avoid piled-up pixels, we extracted a spectrum from the wings of the point-spread function (PSF) for pixels that are between 2".5 and 8 .0 from the centroid. We ignore negligible contributions from other sources in this annulus. An absorbed power law fits the 0.7-8.0 keV spectral data well, with a photon index of $1.2 \pm 0.1, N_{\mathrm{H}}=(8.2 \pm 1.6) \times 10^{21} \mathrm{~cm}^{-2}$, and $\chi_{\nu}^{2}=1.40$ ( $\nu=75$ degrees of freedom [dof]). After a correction for the fraction of the PSF covered $(9 \%)$, the flux is $1.1 \times 10^{-10} \mathrm{ergs}$ $\mathrm{s}^{-1} \mathrm{~cm}^{-2}(0.5-10 \mathrm{keV})$, and the luminosity is $9 \times 10^{35} \mathrm{ergs}$ $\mathrm{s}^{-1}\left(1.1 \times 10^{36} \mathrm{ergs} \mathrm{s}^{-1}\right.$ unabsorbed $)$. The statistical uncertainty is $7 \%$, and that due to PSF uncertainties is an estimated $10 \%$. The flux is roughly 10 times fainter than at the peak and $10^{3}$ times brighter than during quiescence (see below). The source shows no strong variability, with an upper limit of $20 \%$ at $90 \%$ confidence for $100 \mathrm{~s}$ timescales. No narrow features are apparent in the power density spectrum.

The position of CX1 (Pooley et al. 2001) is consistent with that of the candidate optical counterpart V2 in Verbunt et al. (2000). They are 0".46 apart, while the uncertainty in the optical position alone is 0.5 . We conclude that V2 is the secure optical counterpart to the transient in NGC 6440, that CX1 is the quiescent X-ray counterpart, and that the 1998 and 2001 outbursts were from the same object and not from any of the other three or four transient LMXBs in quiescence that are likely to be present in the cluster (Pooley et al. 2001). Most importantly, since type I X-ray bursts were detected from the transient during both 1998 and 2001 outbursts, it is now firmly established that CX1 is a quiescent neutron star (qNS).

NGC 6440 was observed for us in Service Time with the NTT at La Silla 3.5 days after the Chandra observation using the Superb Seeing Imager-2. The seeing was around 1". Images were obtained in the Bessell $R$ and $B$ filters. The analysis of these data is complicated by proper motions of a number of stars in the field. However, the raw images do show V2 to be active again in $B$ at a level comparable to the 1998 outburst.

\section{THE QUIESCENT X-RAY SPECTRUM}

The qNSs exhibit X-ray emission at typical luminosities of $10^{32}-10^{33}$ ergs $\mathrm{s}^{-1}$ (e.g., Asai, Dotani, \& Hoshi 1998; McClintock 2001). A plausible explanation for this emission is ther-

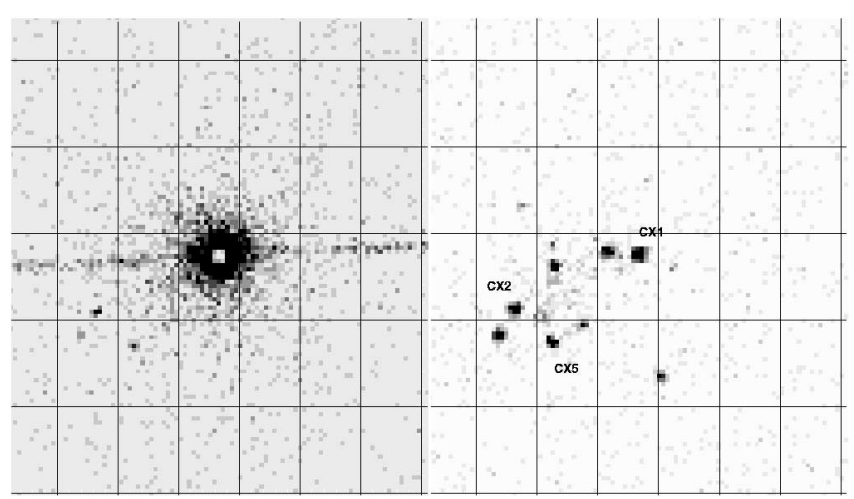

FIG. 1.-Chandra ACIS-S images of NGC 6440 during the transient active state (left; $2.6 \mathrm{ks}$ exposure) and quiescence (right; $23 \mathrm{ks}$ exposure). The grids are fixed to the equatorial coordinate system and defined in the same manner for both cases. Pixels are 0.5 on the side. The left panel shows the bright transient with its large PSF. The hole in the middle of the PSF and the streak are artifacts introduced by the brightness of the transient. The bright transient is coincident with quiescent source "CX1" (Pooley et al. 2001). CX1 is the brightest X-ray source in the globular cluster during quiescence with a $0.5-7 \mathrm{keV}$ unabsorbed luminosity of $(1.7 \pm 0.5) \times 10^{33} \mathrm{ergs} \mathrm{s}^{-1}$.

mal radiation from the atmosphere of a neutron star (NS) that is cooling from the previous outburst (Brown, Bildsten, \& Rutledge 1998). We extracted the spectrum of the transient during quiescence by accumulating all 251 photons within 1 1".5 from the source centroid and applying background corrections as found elsewhere in the image (amounting merely to a 2.3 photons subtraction). To ensure applicability of the $\chi^{2}$ statistic, we rebinned the data so that each channel contains at least 20 photons, except for the highest energy bin, which contains 15 photons. We used CIAO 2.1.3 Science Threads for the background subtraction and response matrix generation. The spectrum was fitted between 0.3 and $6.7 \mathrm{keV}$ (only 2 photons were detected above this range) with a model combining a power law and a NS hydrogen atmosphere model as calculated for the nonmagnetic case by Zavlin, Pavlov, \& Shibanov (1996). Standard absorption by cold interstellar matter of cosmological abundances was applied following Morrison \& McCammon (1983). Due to the limited statistical quality, the fit errors for the atmosphere temperature and radius are reasonably constrained only if the mass of the NS, the distance, the interstellar hydrogen column density $N_{\mathrm{H}}$, and the power-law photon index are fixed. We did so at values of $1.4 M_{\odot}$ (for a "canonical" NS), $8.5 \mathrm{kpc}$ (this is well determined), $N_{\mathrm{H}}=8.2 \times 10^{21} \mathrm{~cm}^{-2}$, and 1.44 (these last two values were determined from the 1998 outburst; in 't Zand et al. 1999), respectively. $N_{\mathrm{H}}$ has an uncertainty of roughly $40 \%$. The only three parameters left free are the NS radius, the atmosphere temperature, and the power-law normalization. The resulting fit is good, with $\chi_{\nu}^{2}=0.758$ (for $\nu=9$ dof). The NS parameters are strongly dependent. The fitted temperature is $92_{-33}^{+172} \mathrm{eV}$, and the radius is $17_{-12}^{+31} \mathrm{~km}(90 \%$ confidence). The radius to an observer at infinity is $19_{-8}^{+29} \mathrm{~km}$. A graph of the spectrum is presented in Figure 2, while the $90 \%$ confidence region in temperature/radius space is presented in Figure 3. If $N_{\mathrm{H}}$ is left free, it converges to $8.2_{-1.3}^{+2.0} \times 10^{21} \mathrm{~cm}^{-2}$, and the range of allowed NS radii surpasses the $100 \mathrm{~km}$ mark. This upper radius margin is also quite sensitive to the power-law index that is poorly constrained by the data.

The inferred radius is consistent with that expected for an NS (e.g., Lattimer \& Prakash 2001). We note that the model implies an intrinsic radius that is $20 \%$ larger if the NS mass is $1.8 M_{\odot}$ instead of $1.4 M_{\odot}$. Fits with some other models are 


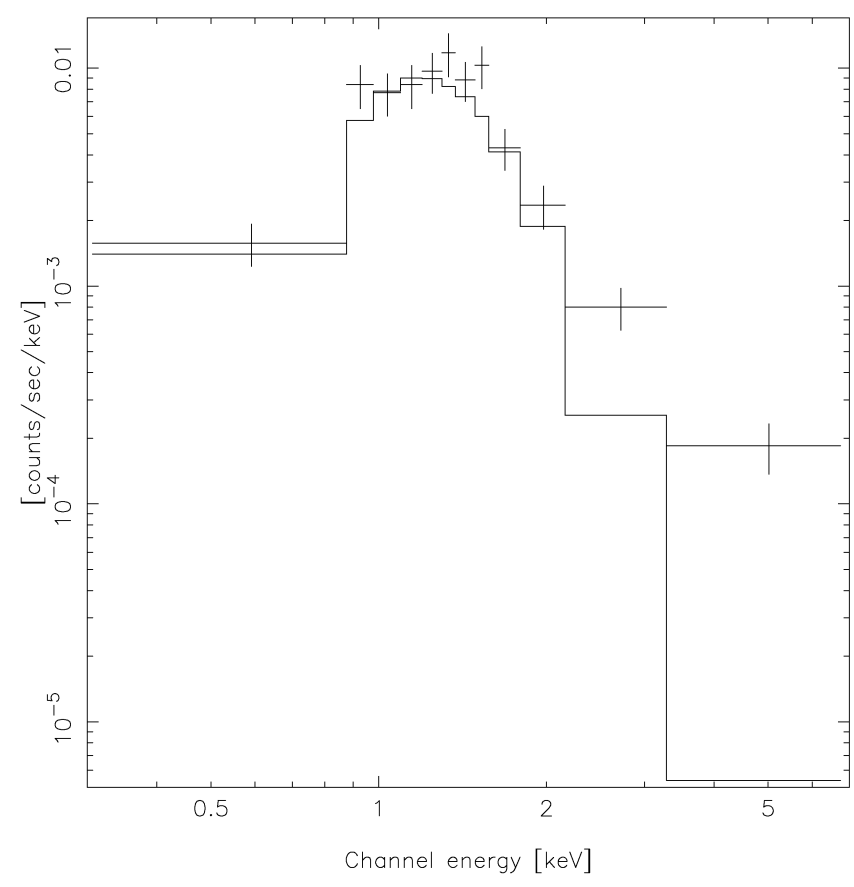

FIG. 2.-Spectrum of the quiescent emission of the transient, as obtained in 2000 July. The crosses refer to the measurements, with the vertical lines indicating the errors, and the histogram refers to the fluxes per channel as predicted by the best-fit NS atmosphere model plus power law, after resetting the power-law contribution to zero to illustrate its impact at high energies. The resetting also illustrates that both spectral components cover fairly distinct photon energies.

nearly as good (Table 1), so the data are not discriminate in that sense. The absorbed $0.5-7 \mathrm{keV}$ flux is $(5.9 \pm 0.4) \times$ $10^{-14} \mathrm{ergs} \mathrm{s}^{-1} \mathrm{~cm}^{-2}$; the unabsorbed flux is $(2.0 \pm 0.6) \times$ $10^{-13} \mathrm{ergs} \mathrm{s}^{-1} \mathrm{~cm}^{-2}$ (the large error reflects the uncertainty in $N_{\mathrm{H}}$ ). The power-law contribution in the latter flux is $20 \% \pm$ $6 \%$ (90\% confidence); this is only $8 \%$ in $0.5-2 \mathrm{keV}$. The bolometric unabsorbed luminosity of the NS atmosphere is $(2.1 \pm 0.8) \times 10^{33} \mathrm{ergs} \mathrm{s}^{-1}$ (the contribution from the uncertainty in the distance is small).

\section{DISCUSSION}

Pooley et al. (2001) find that there are four or five likely quiescent LMXB transients in NGC 6440. Similar conclusions are currently being reached from Chandra observations of other globular clusters such as 47 Tuc (Grindlay et al. 2001a), Liller 1 (Homer et al. 2001), NGC 6652 (Heinke, Edmonds, \& Grindlay 2001), $\omega$ Cen (Rutledge et al. 2001), and NGC 6397 (Grindlay et al. 2001b). Furthermore, for the first time, two bright LMXBs have been identified in a single cluster (M15; White \& Angelini 2001). Our observations show unequivocally that the second and third transient outbursts in NGC 6440 (in 1998 and 2001) are from the same object. Due to a limited positional accuracy of the source of the 1971 outburst, it is not clear whether the same source was responsible for the 1971 and the 1998/2001 outbursts. At first, indirect evidence suggested that the source is different because the duration of 1.5-3 months and the peak flux of 250 mcrab deviate substantially from the values for the 1998 outburst and for the initial phase of the 2001 outburst. However, at the time of this writing, the 2001 outburst shows activity for already 3 months and a peak flux in excess of about 0.2 crab units, according to ASM and WFC measurements.
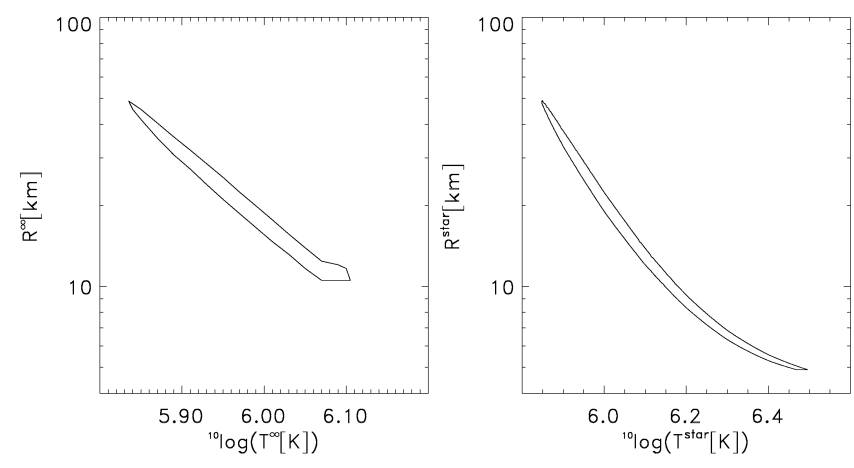

FIG. 3.-Contour plots of the $90 \%$ confidence $(R, T)$-region of an NS atmosphere model fit to the CX1 spectrum during quiescence between 0.3 and $6.7 \mathrm{keV}$, for an observer at infinity (left) or at the NS surface (right). It is assumed that the distance is $8.5 \mathrm{kpc}$ and that $N_{\mathrm{H}}=8.2 \times 10^{21} \mathrm{~cm}^{-2}$. The location of the contour is independent of NS mass for an observer at infinity.

The recurrence time between the two most recent outbursts is unexpectedly short. This may mean that the source has become more active or, alternatively, that earlier outbursts have been missed. It is not uncommon for a previously quiet source to become active in a series of transient outbursts (e.g., GRO J1744-28 and GRO J1655-40). However, the 1998 outburst of the transient in NGC 6440 was so faint and short that similar outbursts may easily have passed undetected. We note that the RXTE ASM and BeppoSAX WFCs became operational only in 1996. In the future, coverage similar to the ASM should resolve this issue.

This is the first time that an optical counterpart has firmly been established for an X-ray transient in a globular cluster. Furthermore, it is the first time that a qNS has been identified without source confusion in a globular cluster. These identifications are important because they present the case of a qNS transient in the optical and X-rays for which an accurate distance is known.

The spectral study of qNSs is foremost interesting, as Brown et al. (1998) pointed out, because it may result in an accurate enough NS radius determination to be able to constrain the equation of state of matter in the star's core. Measuring the radius to an accuracy of about $0.5 \mathrm{~km}$ can exclude $50 \%$ of the proposed equations of state (Lattimer \& Prakash 2001). For NSs in globular clusters as compared with those in the Galactic disk, one source of uncertainty is almost eliminated-the distance. The distance to NGC 6440 is accurate to within 5\%. If other uncertainties (in the spectral parameters) had been at least an order of magnitude lower, the prospects for constraining the equation of state for the transient in NGC 6440 would have

TABLE 1

Goodness of Fit for a Variety of Spectral Models to the 12-Channel Data Between 0.3 and $6.7 \mathrm{keV}$

\begin{tabular}{cllr}
\hline \hline Model & \multicolumn{1}{c}{ Free Parameters } & \multicolumn{1}{c}{$\chi_{v}^{2}$} & \multicolumn{1}{c}{$\nu$} \\
\hline wa (nsa) $\ldots \ldots \ldots \ldots$ & $T$ and $R$ & 2.74 & 10 \\
wa (nsa $+\ldots \ldots) \ldots \ldots$ & $T, R$, and po norm. & 0.758 & 9 \\
wa (bb) $\ldots \ldots \ldots \ldots$. & $T$ and bb norm. & 3.00 & 10 \\
wa (bb + po) $\ldots \ldots \ldots$ & $T$, bb norm., and po norm. & 0.987 & 9 \\
wa (diskbb) $\ldots \ldots \ldots$. & $N_{\mathrm{H}}, T_{\text {in }}$, and norm. & 1.94 & 9 \\
& $T_{\text {in }}$ and norm. & 2.44 & 10 \\
\hline
\end{tabular}

Note. -wa $=$ interstellar absorption following Morrison \& McCammon 1983, nsa = NS H-atmosphere emission according to Zavlin et al. 1996, and po = power law, bb = blackbody, and diskbb $=$ disk blackbody according to Mitsuda et al. 1984. Also, $T=$ temperature, $R=$ radius, and norm. = normalization. 
been good. However, this is not the case. This qNS would have to be observed for a few million seconds with Chandra before it becomes worthwhile to consider this analysis, and that is without considering uncertainties in the spectral models.

Given a peak bolometric luminosity between $4 \times 10^{37}$ and $1.6 \times 10^{38} \mathrm{ergs} \mathrm{s}^{-1}$ (or 50-200 mcrab in 2-10 keV given the broadband spectrum measured by in 't Zand et al. 1999), an $e$-folding decay time between 5 (Verbunt et al. 2000) and 30 days, and assuming that during accretion $100 \%$ of the gravitational potential, energy is released through radiation, the recurrence time of $2.95 \mathrm{yr}$ implies a time-averaged accretion rate of $\langle\dot{M}\rangle \approx 2 \times 10^{-11}$ to $4 \times 10^{-10} M_{\odot} \mathrm{yr}^{-1}$. Following Brown et al. (1998), the quiescent time-averaged luminosity due to thermal emission from the cooling NS is predicted to be between $1 \times 10^{33}$ and $2 \times 10^{34} \mathrm{ergs} \mathrm{s}^{-1}$. Our measurement of $2.1 \times 10^{33} \mathrm{ergs} \mathrm{s}^{-1}$ is consistent with this rough prediction, also if less than $100 \%$ of the liberated potential energy is released as radiation.

We thank Harvey Tananbaum for his willingness to schedule the Chandra observation at short notice, Yousaf Butt for help in planning the Chandra observation, Olivier Hainaut for carrying out the optical observations, Rob van der Meer for help in the ACIS data analysis, Slava Zavlin and George Pavlov for providing their neutron star atmosphere models in electronic form, and Jaap Schuurmans for software support. J. J. M. in 't Zand acknowledges financial support from the Netherlands Organization for Scientific Research (NWO), M. H. van Kerkwijk through a fellowship of the Royal Netherlands Academy of Sciences (KNAW), D. Pooley through a National Science Foundation Graduate Fellowship, W. H. G. Lewin from NASA, and R. Wijnands from NASA through a Chandra Fellowship.

\section{REFERENCES}

Asai, K., Dotani, T., \& Hoshi, R. 1998, PASJ, 50, 611

Bradt, H., Levine, A., Remillard, R., \& Smith, D. A. 2001, in Proc. X-Ray Astronomy '99: Stellar Endpoints, AGN, and the Diffuse Background, ed. G. Malaguti, G. Palumbo, \& N. White (New York: AIP), in press (astro$\mathrm{ph} / 0003438)$

Brown, E. F., Bildsten, L., \& Rutledge, R. E. 1998, ApJ, 504, L95

Forman, W., Jones, C., \& Tananbaum, H. 1976, ApJ, 207, L25

Grindlay, J. E., Heinke, C., Edmonds, P. D., \& Murray, S. S. 2001a, Science, 292,2290

- 2001b, ApJL, submitted (astro-ph/0108265)

Guainazzi, M., Parmar, A. N., \& Oosterbroek, T. 1999, A\&A, 349, 819

Heinke, C. O., Edmonds, P. D., \& Grindlay, J. E. 2001, ApJ, 562, 363

Homer, L., Deutsch, E. W., Anderson, S. F., \& Margon, B. 2001, AJ, in press (astro-ph/0106140)

in 't Zand, J. J. M. 2001, in Proc. Fourth INTEGRAL Workshop, Exploring the Gamma-Ray Universe, ed. A. Gimenez, V. Reglero, \& C. Winkler (Noordwijk: ESA), in press (astro-ph/0104299)

in 't Zand, J. J. M., Pooley, D., Lewin, W. H. G., van Kerkwijk, M. H., \& Verbunt, F. 2001, Astron. Telegram 74

in 't Zand, J. J. M., et al. 1999, A\&A, 345, 100

Jager, R., et al. 1997, A\&AS, 125, 557

Lattimer, J. M., \& Prakash, M. 2001, ApJ, 550, 426
Levine, A., Bradt, H., Cui, W., Jernigan, J. G., Morgan, E. H., Remillard, R., Shirley, R. E., \& Smith, D. A. 1996, ApJ, 469, L33

Markert, T. H., Backman, D. E., Canizares, C. R., Clark, G. W., \& Levine, A. M. 1975, Nature, 257, 32

McClintock, J. E. 2001, in Proc. Jan van Paradijs Memorial Symp., From XRay Binaries to Gamma-Ray Bursts, ed. E. P. J. van de Heuvel, L. Kaper, \& E. Rol (San Francisco: ASP), in press (astro-ph//0109423)

Mitsuda, K., et al. 1984, PASJ, 36, 741

Morrison, R., \& McCammon, D. 1983, ApJ, 270, 119

Ortolani, S., Barbuy, B., \& Bica, E. 1994, A\&AS, 108, 653

Pooley, D., et al. 2001, ApJ, submitted (astro-ph/0111212)

Rutledge, R. E., Bildsten, L., Brown, E. F., Pavlov, G., \& Zavlin, V. 2001, ApJ, submitted (astro-ph/0105405)

Sidoli, L., Parmar, A. N., Oosterbroek, T., Stella, L., Verbunt, F., Masetti, N., \& Dal Fiume, D. 2001, A\&A, 368, 451

Verbunt, F., van Kerkwijk, M. H., in 't Zand, J. J. M., \& Heise, J. 2000, A\&A, 359,960

Weisskopf, M. C., O’dell, S. L., \& van Speybroeck, L. P. 1996, Proc. SPIE, 2805,2

White, N. E., \& Angelini, L. 2001, ApJ, 561, L101

Zavlin, V. E., Pavlov, G. G., \& Shibanov, Yu. A. 1996, A\&A, 315, 141 